\title{
Energy and information in Hodgkin-Huxley neurons
}

\author{
A. Moujahid, A. d'Anjou, and F. J. Torrealdea \\ Department of Computer Science, University of the Basque Country, ES-20018 San Sebastian, Spain \\ F. Torrealdea \\ UCL Institute of Neurology, London WC1N 3BG, United Kingdom \\ (Received 9 August 2010; revised manuscript received 21 January 2011; published 21 March 2011)
}

\begin{abstract}
The generation of spikes by neurons is energetically a costly process and the evaluation of the metabolic energy required to maintain the signaling activity of neurons a challenge of practical interest. Neuron models are frequently used to represent the dynamics of real neurons but hardly ever to evaluate the electrochemical energy required to maintain that dynamics. This paper discusses the interpretation of a Hodgkin-Huxley circuit as an energy model for real biological neurons and uses it to evaluate the consumption of metabolic energy in the transmission of information between neurons coupled by electrical synapses, i.e., gap junctions. We show that for a single postsynaptic neuron maximum energy efficiency, measured in bits of mutual information per molecule of adenosine triphosphate (ATP) consumed, requires maximum energy consumption. For groups of parallel postsynaptic neurons we determine values of the synaptic conductance at which the energy efficiency of the transmission presents clear maxima at relatively very low values of metabolic energy consumption. Contrary to what could be expected, the best performance occurs at a low energy cost.
\end{abstract}

DOI: 10.1103/PhysRevE.83.031912

PACS number(s): 87.19.11, 87.19.1g, 87.19.1y, 87.18.Sn

\section{INTRODUCTION}

An action potential is a sudden increase in the membrane potential of a neuron followed by a subsequent rapid decrease that produces a traveling wave with a characteristic spike shape. The variation in the membrane potential is produced by the flux of different ion species across the cell membrane, which precise regulation dictates the shape of the spike. Ion pumps consume adenosine triphosphate (ATP) molecules to extrude ions through the cell membrane against their gradient concentrations. If the rate of generation of ATP supplies is not enough to satisfy the demand of the ion pumps, the refractory period of the neuron will increase and the information transmitted will be altered. On a global scale the metabolic energy required to maintain neural activity is very high [1-3], and a large fraction of this total energy is expended in the generation of firing sequences of action potentials [1].

Estimating the energy consumed by a neuron during an action potential is usually done by recording some action potentials and theoretically calculating the number of sodium ions entering the cell required to produce the same membrane depolarization. This calculated $\mathrm{Na}^{+}$provides an estimate of the number of pump cycles, or ATP molecules, that the pump will need to reestablish the resting state of the neuron. However, this estimation is the subject of controversy [4]. Inward $\mathrm{Na}^{+}$ and outward $\mathrm{K}^{+}$overlap during the action potential generation, introducing an uncertainty in the calculation of sodium ions up to a factor of 4 [1,5-7]. Recent work with model neurons shows that the energy cost of an action potential varies up to 22-fold through differences in overlap and height of the spikes [8]. In addition, the transmembrane enzyme ATPase moves per cycle three $\mathrm{Na}^{+}$ions outside the cell and only two $\mathrm{K}^{+}$ions inside. This unbalanced electric exchange generates controversy about whether to use one third or half the number of sodium ions to estimate the number of pump cycles $[9,10]$.

Our approach to estimating the energy is different and requires no hypothesis about overlapping or ion counting. This paper studies in detail the consumption of energy by neurons whose signaling activity is represented by the Hodgkin-Huxley equations [11]. Be it in its original formulation or with appropriate adaptations [12-15], the Hodgkin-Huxley precise representation of the dynamics of the squid giant axon is the most frequently used model in the study of actual neurons. We consider that if the Hodgkin-Huxley electric circuit is a valid tool to investigate the dynamics and electrical properties of neurons, it will be also valid to analyze the actual energy the neurons demand to produce that dynamics.

The evaluation of the cost of information requires knowledge of both the metabolic energy cost and the amount of information transmitted by a train of action potentials. Shannon's information theory [16] provides a framework to quantify the amount of information that neurons can convey during their signaling activity.

In Sec. II of this paper we analyze the electrochemical energy of an isolated Hodgkin-Huxley neuron and calculate its energy consumption. After studying the metabolic energy cost of an isolated neuron we analyze the balance of energy when two neurons are coupled by an electrical synapse. The underlying reason to do it is that a way to propagate information through a channel of neurons is via synchronization of the postsynaptic neuron to the train of action potentials it receives from its presynaptic cell. For instance, the flow of visual signals in the retina requires electrical gap junctions between AII amacrine cells and ON-cone bipolar cells [17], and temporally precise synchronization of subthreshold membrane potential fluctuations has been demonstrated [18].

We have studied groups of postsynaptic neurons coupled unidirectionally by electrical synapses to a single presynaptic neuron and investigated how the energy efficiency of the information transmission depends both on the values of the synaptic conductance and on the size of the receiving group of neurons. We have found that when the receptor is a single neuron the increase in mutual information with the coupling conductance is faster than the corresponding increase in metabolic energy 
consumption. This results in a monotonic increase of the energy efficiency with the coupling conductance. For a single receiving neuron there is no compromise; maximum efficiency occurs at maximum energy consumption. On the other hand, for groups of receiving neurons there are values of the synaptic conductance, with relatively low consumption, that optimize the ratio of mutual information to metabolic energy consumption. For groups of receiving neurons maximum efficiency does not require maximum energy consumption. Section III presents computational results for four different sized groups of neurons, which include information entropies and mutual information, metabolic energy consumptions, and information-to-energy ratios. Finally in Sec. IV we discuss the results obtained in the paper.

\section{ENERGY OF THE HODGKIN-HUXLEY NEURON}

The Hodgkin-Huxley model of the squid giant axon without action potential propagation [11], which we take in this work as representative of the dynamics of a generic neuron, obeys the following differential equations:

$$
\begin{gathered}
C \dot{V}=-i_{\mathrm{Na}}-i_{\mathrm{K}}-i_{l}+I, \\
\dot{m}=\alpha_{m}(V)(1-m)-\beta_{m}(V) m, \\
\dot{n}=\alpha_{n}(V)(1-n)-\beta_{n}(V) n, \\
\dot{h}=\alpha_{h}(V)(1-h)-\beta_{h}(V) h,
\end{gathered}
$$

where $V$ is the membrane potential in $\mathrm{mV}, C$ is the membrane capacitance in $\mu \mathrm{F}, I$ is the total membrane current density in $\mu \mathrm{A} / \mathrm{cm}^{2}$ and $m, n$, and $h$ are dimensionless variables that represent, respectively, the proportion of sodium-activating molecules on the inside of the membrane, the proportion of potassium-activating particles on the inside, and the proportion of sodium-inactivating molecules on the outside [11].

The ion currents of sodium, potassium, and leakage (mainly chloride), $i_{\mathrm{Na}}, i_{\mathrm{K}}$, and $i_{l}$, respectively, are given by

$$
\begin{gathered}
i_{\mathrm{Na}}=g_{\mathrm{Na}} m^{3} h\left(V-E_{\mathrm{Na}}\right), \\
i_{\mathrm{K}}=g_{\mathrm{K}} n^{4}\left(V-E_{\mathrm{K}}\right), \\
i_{l}=g_{l}\left(V-E_{l}\right),
\end{gathered}
$$

where $g_{\mathrm{Na}}, g_{\mathrm{K}}$, and $g_{l}$ are the maximum possible values of the conductances of the respective ion channels and $E_{\mathrm{Na}}, E_{\mathrm{K}}$, and $E_{l}$ the Nernst potentials of the ions in the resting state of the neuron. In this work we have used for these parameters the standard constant values given in Table I [19]. The variables over time alpha and beta functions are

$$
\begin{gathered}
\alpha_{m}(V)=(2.5-0.1 V) /[\exp (2.5-0.1 V)-1], \\
\beta_{m}(V)=4 \exp (-V / 18), \\
\alpha_{n}(V)=(0.1-0.01 V) /[\exp (1-0.1 V)-1], \\
\beta_{n}(V)=0.125 \exp (-V / 80), \\
\alpha_{h}(V)=0.07 \exp (-V / 20), \\
\beta_{h}(V)=1 /[\exp (3-0.1 V)+1] .
\end{gathered}
$$

\section{A. Energy balance of the electrical circuit}

The Hodgkin-Huxley equations express the dynamics of an electrical circuit where $g_{\mathrm{Na}}, g_{\mathrm{K}}$, and $g_{l}$ are the maximum values
TABLE I. Parameters of the Hodgkin-Huxley equations. The membrane capacity is $C=1 \mu \mathrm{F} / \mathrm{cm}^{2}$. The voltage scale is shifted so that the resting potential vanishes.

\begin{tabular}{lcc}
\hline \hline$x$ & $g_{x}\left(\mathrm{mS} / \mathrm{cm}^{2}\right)$ & $E_{x}(\mathrm{mV})$ \\
\hline $\mathrm{Na}$ & 120 & 115 \\
$\mathrm{~K}$ & 36 & -12 \\
1 & 0.3 & 10.6 \\
\hline \hline
\end{tabular}

of the respective variable conductances, and batteries stand for the Nernst potentials of their corresponding ions. If $V$ is the membrane potential, the total electrical energy accumulated in the circuit at a given moment in time is

$$
H(t)=\frac{1}{2} C V^{2}+H_{\mathrm{Na}}+H_{\mathrm{K}}+H_{l},
$$

where the first term in the summation represents the electrical energy accumulated in the capacitor, and the other three terms are the respective energies in the batteries. The electrochemical energy accumulated in the batteries is unknown. In the model it is potentially unlimited as we do not contemplate the exhaustion of the batteries. In the real neuron, the intake of nutrients prevents the exhaustion of the ion pumps. Nevertheless, the rate of electrical energy provided to the circuit by a battery is known to be the electrical current through the battery times its electromotive force. Thus, the total derivative with respect to time of the above energy will be

$$
\dot{H}(t)=C V \dot{V}+i_{\mathrm{Na}} E_{\mathrm{Na}}+i_{\mathrm{K}} E_{\mathrm{K}}+i_{l} E_{l} .
$$

Let $I$ stand for the total external current that, one way or another, is injected through the membrane. According to the first equation in Eq. (1) we have

$$
C \dot{V}=I-i_{\mathrm{Na}}-i_{\mathrm{K}}-i_{l},
$$

which substituted in Eq. (4) gives

$$
\dot{H}=V I-i_{\mathrm{Na}}\left(V-E_{\mathrm{Na}}\right)-i_{\mathrm{K}}\left(V-E_{\mathrm{K}}\right)-i_{l}\left(V-E_{l}\right) .
$$

If we substitute Eq. (2) for the ion currents, we have for the energy rate in the circuit

$$
\begin{aligned}
\dot{H}= & V I-g_{\mathrm{Na}} m^{3} h\left(V-E_{\mathrm{Na}}\right)^{2}-g_{\mathrm{K}} n^{4}\left(V-E_{\mathrm{K}}\right)^{2} \\
& -g_{l}\left(V-E_{l}\right)^{2},
\end{aligned}
$$

which provides the total derivative of the electrochemical energy in the neuron as a function of its state variables. The first term in the right-hand summation represents the electrical power given to the neuron via the different junctions reaching the neuron, and the other three terms of the summation represent the energy per second consumed by the ion channels. As the firing rate depends on the total current $I$ the average metabolic consumption of the Hodgkin-Huxley neuron as a function of the firing rate can be calculated simply evaluating Eq. (5) at different values of the external current $I$. The following two sections present results of these calculations.

\section{Energy consumption rate in the generation of action potentials}

A train of action potentials has been generated integrating the model neuron equations at a value of the total current $I=6.9 \mu \mathrm{A} / \mathrm{cm}^{2}$. At this value of the external current the neuron fires tonically at about $57 \mathrm{~Hz}$. Figure 1(a) shows a 


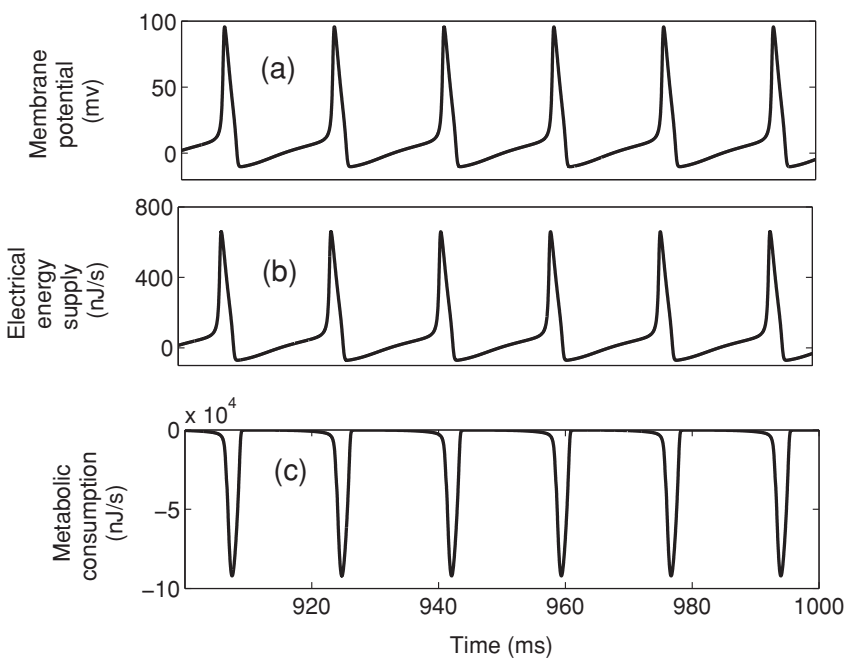

FIG. 1. (a) Action potentials generated at $I=6.9 \mathrm{nA}$. (b) Energy supplied per second at the synaptic junction. (c) Energy dissipated per second at the ion channels.

sequence of the generated train of actions potentials. Time is in milliseconds, and the membrane potential changes from a few millivolts below zero in its polarized state to $100 \mathrm{mV}$ after depolarization. Figure 1(b) depicts against time the electrical energy rate corresponding to the first term $V I$ in the right-hand side of Eq. (5). It is positive on average with peaks reaching about $600 \mathrm{~nJ} / \mathrm{s}$. It supposes a net contribution of energy to the neuron whose origin depends on the origin of current $I$. In the case of experimental electrode recordings, for instance, the electrode-clamping device would be the source of energy. In case $I$ comes from the synapse this contribution of energy represents energy supplied per second at the synaptic junction. In Fig. 1(c) the total metabolic energy consumption of the neuron is represented. The figure depicts the total contribution to the energy derivative of the three last terms of Eq. (5). Note that it is negative and represents the instantaneous total consumption of energy per second at the ion channels. This electrochemical energy consumption reaches peaks near $100000 \mathrm{~nJ} / \mathrm{s}$ and is much greater than the energy supplied by the $V I$ term. Basically, this rate of energy must be replenished by the ion pumps and metabolically supplied by hydrolysis of ATP molecules in order to maintain the neuron's activity.

In order to analyze in more detail the ion currents involved in the generation of action potentials and their contributions to the energy consumption, Fig. 2(a) shows currents of sodium, potassium, and chloride ions corresponding to one particular action potential of the previously described train and Fig. 2(b) the electrochemical energy consumption associated with each of the ions. The currents are generated in response to a change in the respective ion conductances. The sodium current is negative but has been depicted with a positive sign for a better appreciation of the great extent that it overlaps with the potassium current. Both sodium and potassium currents reach a peak around $700 \mu \mathrm{A} / \mathrm{cm}^{2}$; the leaking current is much smaller. Note that as sodium and potassium currents both carry positive charges but move in opposite directions on the cell membrane, they neutralize each other to the extent of their mutual overlap, and the net membrane current is much smaller. Nevertheless,
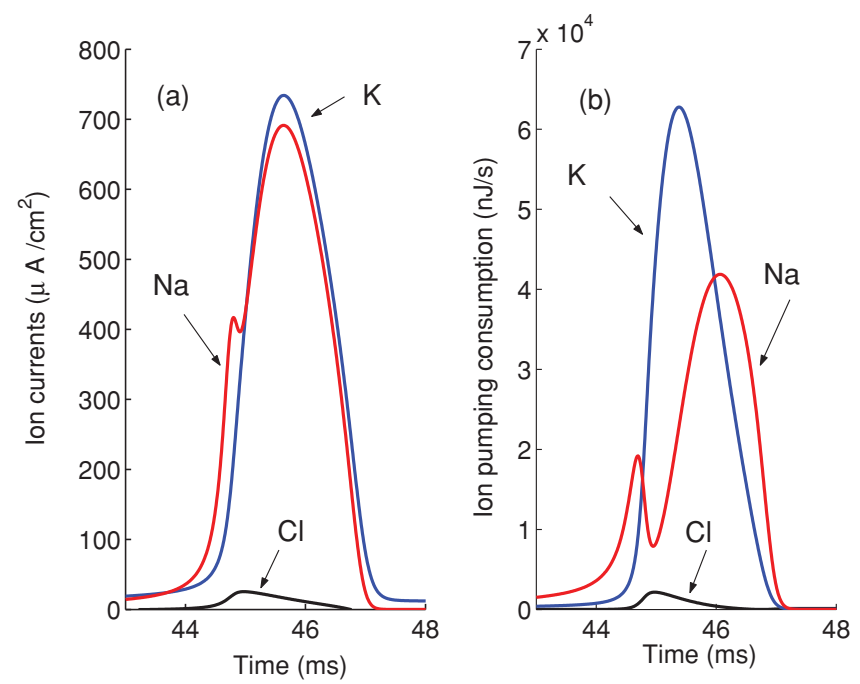

FIG. 2. (Color online) (a) Currents of sodium, potassium, and chloride ions at an action potential. Sodium current is negative but has been represented with a positive sign. (b) Electrochemical energy consumption corresponding to each of the ion currents. Although depicted as positive, the three are negative. Action potentials have been generated at $I=6.9 \mu \mathrm{A} / \mathrm{cm}^{2}$.

the actual movement of ions takes place and involves energy consumption whether the ions collaborate to the establishment of the membrane potential or not. The total number of $\mathrm{Na}^{+}$ ions that permeate the membrane during the action potential is proportional to the area under the sodium current curve in the figure. As said in Sec. I, this total number of $\mathrm{Na}^{+}$ions is used to estimate the number of ATP molecules that the ion pump will need to reestablish the ion concentrations. Figure 2(b) shows the electrochemical energy balance associated with each of the ion currents. The three ion channels contribute negatively to the energy derivative in Eq. (5), which has been represented as a positive energy consumption. Note that in our approach the metabolic consumption of the neuron in generating one action potential is directly the sum of these three components. Reestablishing the concentrations of each of the three ions $\mathrm{Na}^{+}, \mathrm{K}^{+}$, and $\mathrm{Cl}^{+}$requires energy, and the approach is independent of whether they move together using one ATP molecule per pump cycle or not.

\section{Average energy consumption as function of the external current}

The instantaneous energy consumption of a neuron is not of direct interest as it continuously changes during the time span of the action potential generation. Average values per unit time are more useful. To investigate the degree of change of the average metabolic consumption of a neuron associated with its firing rate we have averaged, over long periods of time of 750 seconds, ion currents and ion consumptions at different values of the external current $I$. Figure 3(a) shows the average values of the sodium and potassium currents for a long train of action potentials. For values of the external current $I$ between $I=4$ and $6.2 \mu \mathrm{A} / \mathrm{cm}^{2}$ the neuron is at its resting state without firing. In this region, the small conductance of the sodium channels maintains this current at very slow values. The potassium current is slightly larger, compensating the leakage and the 

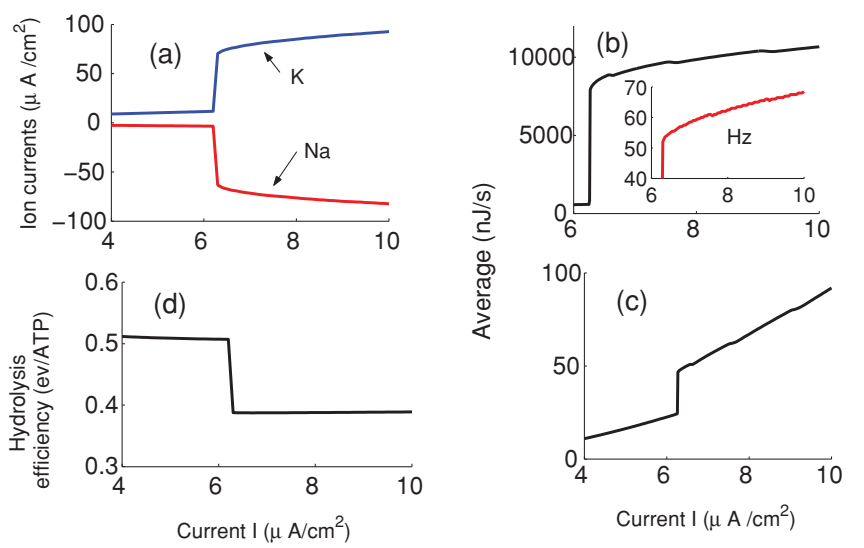

FIG. 3. (Color online) (a) Average of sodium and potassium currents on a long train of action potentials at different values of the external applied current $I$. (b) Average total consumption of energy per second as a function of the applied current $I$. The inset displays the corresponding firing rates in hertz. (c) Average energy per second supplied to the neuron through the injection site. Its increase with current $I$ is linear with different slopes in the quiescent and firing regimes. It is always much less than the dissipation at the ionic channels. Action potentials have been generated at $I=6.9 \mu \mathrm{A} / \mathrm{cm}^{2}$. (d) Ratio of the total energy consumption to one third of the number of sodium ions. The ratio is expressed in electronvolts per ATP and represents the efficiency, in the model, of the ATP hydrolysis measured as the free energy that is provided by the hydrolysis of one molecule of ATP.

external current. For larger values of the external current $I$ the neuron acquires a tonic firing regimen of increasing rate. The average values of the sodium and potassium currents clearly detect the transition and increase with the increasing firing rate. In Fig. 3(b) the average of the total energy consumption per unit time, all three channels, as a function of the applied current $I$ is shown. The vertical sudden increase in energy consumption clearly reveals the transition from a quiescent state without firing at low values of the current $I$ to the firing regime induced by larger values of the applied current. The higher demand of energy associated with the generation of spikes is clearly visible; for instance, at $I=6.9 \mu \mathrm{A} / \mathrm{cm}^{2}$, which is the value used to generate the train of action potentials previously analyzed, the average metabolic consumption is about $9000 \mathrm{~nJ} / \mathrm{s}$. This consumption must be replenished by metabolic ATP supply. Firing frequencies corresponding to different values of current $I$ are displayed in the inset. Figure 3(c) shows the average energy per second supplied to the neuron by the $V I$ term. Its increase with current $I$ is linear with different slopes for the quiescent and firing regimes. It is always much lower than the dissipation at the ionic channels. In Fig. 3(d) the ratio of the total energy consumption to one third of the number of sodium ions through the membrane is shown. The ratio is expressed in electronvolts per ATP and represents the efficiency of the ATP hydrolysis measured as the free energy that is provided, in the model, by the hydrolysis of one molecule of ATP. As can be seen in the figure, this ratio is constant for the whole range of values of $I$ between $I=6.2$ and $10 \mu \mathrm{A} / \mathrm{cm}^{2}$; that is, it is independent of the firing rate. Thus, our results show that during firing the pump demands each ATP molecule $0.39 \mathrm{eV}$, which is in excellent agreement with the $0.37 \mathrm{eV}$ estimated in the literature [20] for one pumping cycle that moves two potassium ions in and three sodium ions out of the cell. This result also agrees with the reported data of $0.43 \mathrm{eV}$ of free energy that one ATP molecule can liberate [21], which would imply a high efficiency of ATP usage with only $10 \%$ of its liberated free energy lost in thermal energy. On the other hand, the required efficiency in the model for the ATP hydrolysis of $0.51 \mathrm{eV}$, when the neuron is at its resting state, is slightly larger than the energy an ATP molecule can liberate. This result points in the direction that the Hodgkin-Huxley model underestimates the number of sodium ions when the neuron is at rest.

\section{B. Energy balance of two electrically coupled neurons}

Gap junction channels permit the intracellular electrical potentials of two neurons to directly connect together and are usually referred to as electrical synapses. They are frequent when two or more neurons are coupled together and play an important role in the synchronization of cellular events. In particular, they are efficient in transmitting information and in synchronizing the information of groups of neurons [22]. Although symmetry is an expected property for an electrical synapse, asymmetric gap junctions have also been reported in the literature. In the retina, for instance, there is evidence that signal transmission is more effective in the direction from AII amacrine cells to ON-cone bipolar cells than in the other direction [18]. This functional rectification can be explained by a corresponding difference in membrane input resistance between the two cell types. In this work, in order to preserve a definite direction for the information transmission, we have studied completely asymmetric unidirectional gap junctions.

\section{Unidirectional coupling}

Gap junction channels can directly connect two neurons' somata or form contact sites at the tips of neurites. In any case, we consider that the contact sites do not contribute to the capacitance of the circuit [22]. Both neurons obey the set of Eq. (1) with the addition of a coupling affecting the postsynaptic neuron. The membrane current density of the presynaptic neuron has been represented by $I_{\text {stimulus }}(t)$, a current supposedly induced by an excitatory external stimulus. It has been modeled by a Gaussian noise of mean $0 \mu \mathrm{A}$ and variance $9 \mu \mathrm{A}$. The stimulus input parameters mean and variance of the Gaussian noise have been experimentally chosen so that they give rise to sufficient variability in the spike train of the presynaptic neuron. This entropic train of spikes remains statistically unchanged throughout our work and has been used as the source of information. The postsynaptic neuron, supposedly an interneuron, is exposed to a total noise current $I_{\text {noise }}$ modeled by a Gaussian noise of mean $0 \mu \mathrm{A}$ and variance $1 \mu \mathrm{A}$. This noise can be supposed the mean of erratic signals arriving at the postsynaptic neuron from other synapses that we do not specifically consider. This noise has not been introduced in the presynaptic neuron as there is no special 


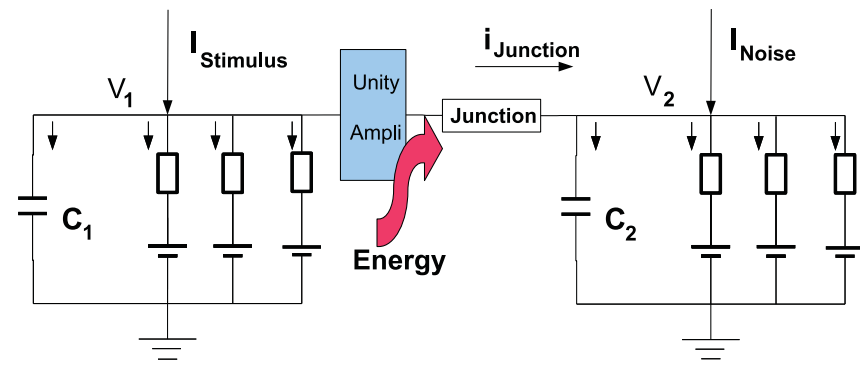

FIG. 4. (Color online) Electrical unidirectional coupling; HH electrical circuit.

difficulty in considering it part of the stimulus current. The two equations for the membrane potentials of each neuron are

$$
\begin{gathered}
C_{1} \dot{V}_{1}=I_{\text {stimulus }}(t)-i_{1 N_{a}}-i_{1 K}-i_{1 l}, \\
C_{2} \dot{V}_{2}=I_{\text {noise }}(t)-i_{2 N_{a}}-i_{2 K}-i_{2 l}+I_{\text {Junction }} .
\end{gathered}
$$

The current through the junction is $I_{\text {Junction }}=k\left(V_{1}-V_{2}\right)$, where the parameter $k$ is the conductance of the gap junction, or coupling strength, in $\mathrm{mS} / \mathrm{cm}^{2}$. The current $I_{\text {stimulus }}(t)$ represents the variable current stimulating the presynaptic neuron that carries information to be transmitted by the neurons, and $I_{\text {noise }}(t)$ stands for the total noise current arriving at the postsynaptic neuron through other synapses.

Thus, in a unidirectional coupling no flow of current is allowed from or to the presynaptic neuron. Nevertheless, a junction current $k\left(V_{1}-V_{2}\right)$ must still flow into the receiving neuron through the electrical junction. Figure 4 shows a possible electrical circuit that implements a unidirectional coupling between two Hodgkin-Huxley neurons. The difference in electrical potential in the union is maintained by an amplifier of unity gain with very large entrance impedance that keeps the first neuron energetically independent of the second one. The energy that the postsynaptic neuron needs at the junction is provided by the amplifier. The amplifier provides a current $I_{\text {Junction }}$ at a voltage $V_{1}$; that is, a power $V_{1} I_{\text {Junction. }}$. The electrical energy in the circuit corresponding to the postsynaptic neuron, which is the only one affected by the coupling, is now

$$
H_{2}(t)=\frac{1}{2} C_{2} V_{2}^{2}+H_{2 \mathrm{Na}}+H_{2 \mathrm{~K}}+H_{2 l}+H_{\text {Amplifier }},
$$

which is the summation of the accumulated electrical energy in the capacitor plus the electrochemical energy in the three batteries plus the energy that is available in the amplifier. The total energy derivative in the second neuron is given by

$$
\begin{aligned}
\dot{H}_{2}= & V_{2} I_{\text {noise }}-g_{2 \mathrm{Na}} m_{2}^{3} h_{2}\left(V_{2}-E_{2 \mathrm{Na}}\right)^{2} \\
& -g_{2 \mathrm{~K}} n_{2}^{4}\left(V_{2}-E_{2 \mathrm{~K}}\right)^{2}-g_{2 l}\left(V_{2}-E_{2 l}\right)^{2} \\
& +k V_{2}\left(V_{1}-V_{2}\right)+k V_{1}\left(V_{1}-V_{2}\right) .
\end{aligned}
$$

The joint contribution of the three ion channels on the right-hand side of this derivative is used throughout the paper to compute the metabolic consumption of the postsynaptic neuron. The last two terms represent the balance of energy in the junction, which correspond to the energy consumed at the postsynaptic site of the junction plus the energy contributed by the amplifier. Note that this net consumption of metabolic energy in the junction is not necessarily negative, and consequently the junction itself could be an active supplier of energy. In any case, the energy does not come from the signaling neuron, which provides information but not energy.

\section{Computational results for the unidirectional coupling}

The average metabolic energy consumptions, as a function of the coupling conductance $k$, for two neurons coupled unidirectionally are shown in Fig. 5. The metabolic energy consumption has been computed with the contribution of all three ion terms of Eq. (7) for the postsynaptic neuron and with their equivalent terms for the presynaptic one. As spike trains are noisy, convergence of some stochastic characteristics, such as the energy consumption rate, requires averages over long periods of time. To perform this calculation the average has been taken over a train of action potentials of 750 seconds length. Curves are depicted against the coupling conductance $k$, and we have reversed the actual negative sign of the energy derivative in the representation. As the coupling is unidirectional, the conductance of the junction has no effect on the presynaptic neuron, and its average metabolic energy consumption remains constant near $9000 \mathrm{~nJ} / \mathrm{s}$. The postsynaptic neuron starts at $k=0 \mathrm{mS} / \mathrm{cm}^{2}$ with a consumption about $500 \mathrm{~nJ} / \mathrm{s}$ that corresponds to its quiescent state altered from time to time by a random excitation. As the strength of the coupling increases, the metabolic consumption progressively increases until it reaches nearly the same levels of consumption of the presynaptic neuron at values of the coupling strength about $k=0.1 \mathrm{mS} / \mathrm{cm}^{2}$. At this point the firing frequency of the postsynaptic neuron has reached that of the presynaptic one. Average firing frequencies are shown in the inset.

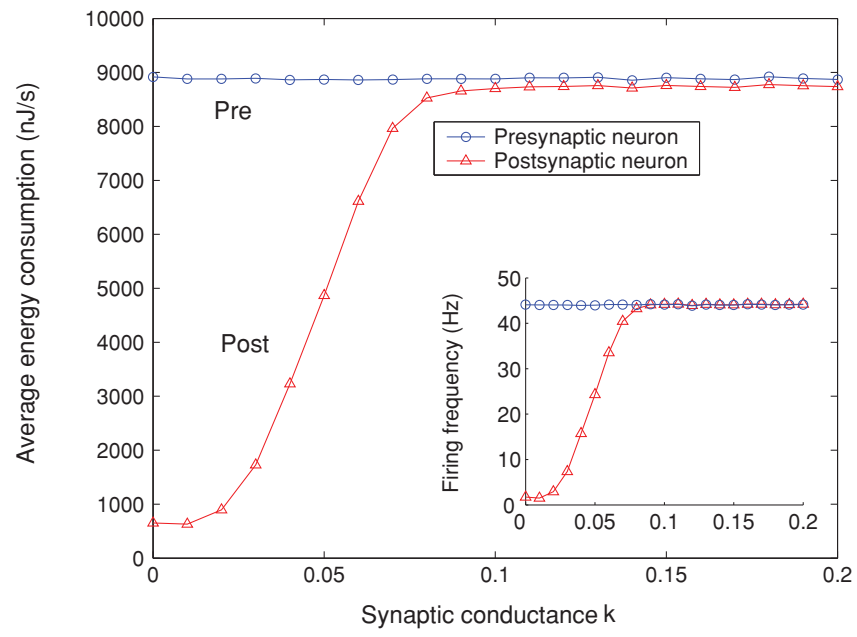

FIG. 5. (Color online) Average metabolic energy consumption of the ionic channels of the sending and receiving neurons at different values of the synaptic conductance $k$. The stimulus current $I_{\text {stimulus }}(t)$ of the presynaptic neuron, supposedly induced by an excitatory external stimulus, has been modeled by a Gaussian noise of mean $0 \mu \mathrm{A}$ and variance $9 \mu \mathrm{A}$. The postsynaptic neuron, supposedly an interneuron in charge of transmitting the information, is exposed to a total noise current $I_{\text {noise }}$, supposedly arriving at it through other synapses that we do not specifically consider, modeled by a Gaussian noise of mean $0 \mu \mathrm{A}$ and variance $1 \mu \mathrm{A}$. The inset shows average firing frequencies as function of the conductance $k$ for both neurons. Unidirectional coupling. 


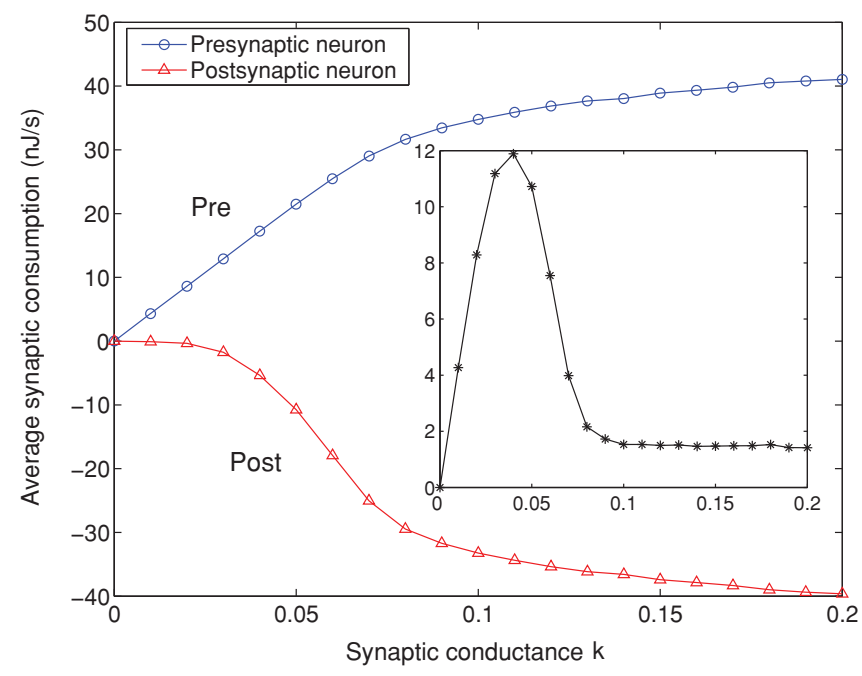

FIG. 6. (Color online) Average energy per second supplied from the extracellular medium and average dissipation to the extracellular medium at the postsynaptic site at different values of the synaptic conductance $k$. In the inset is shown the net energy derivative at the junction, which is positive; that is, there is a net income of energy from the synapse. Unidirectional coupling.

We have also analyzed the balance of energy in the unidirectional synaptic junction. As there cannot be any flow of energy between the neurons, the postsynaptic neuron can exchange ions and energy only with the extracellular milieu, although under control by the presynaptic neuron. In Fig. 6 the two components of the synaptic metabolic energy, two last terms of Eq. (7), have been depicted against the coupling conductance $k$. The first term is on average always negative and represents dissipation of energy at the junction. The second term is always positive, energy delivered to the neuron by the amplifier, and represents energy entering the junction from the external milieu. Thus, the postsynaptic neuron both dissipates and takes energy at the junction site. The inset in Fig. 6 shows the joint contribution of both components. As can be appreciated the balance is positive for the neuron; that is, there is a net, although small, income of energy to the neuron through the synaptic site with a sharp maximum of this incoming energy at above $k=0.04 \mathrm{mS} / \mathrm{cm}^{2}$. As we can see in Fig. 5, and more clearly in Sec. III for a group of postsynaptic neurons (see Fig. 10), it seems to be an inflexion point in the metabolic energy consumption of the postsynaptic neuron at this value of the coupling conductance. The relevance of this fact is unknown to us.

\section{GROUPS OF POSTSYNAPTIC NEURONS: ENERGY AND INFORMATION}

Groups of uncoupled neurons processing identical input can behave as a more robust computational unit against random noise $[23,24]$. In this section we study networks composed of Hodgkin-Huxley neurons such as the one represented in the schematic diagram of Fig. 7. The computational setting corresponds to one presynaptic neuron that acts as a generator of information via spike trains $V_{S}(t)$, which are transmitted through unidirectional electrical gap junctions to a group

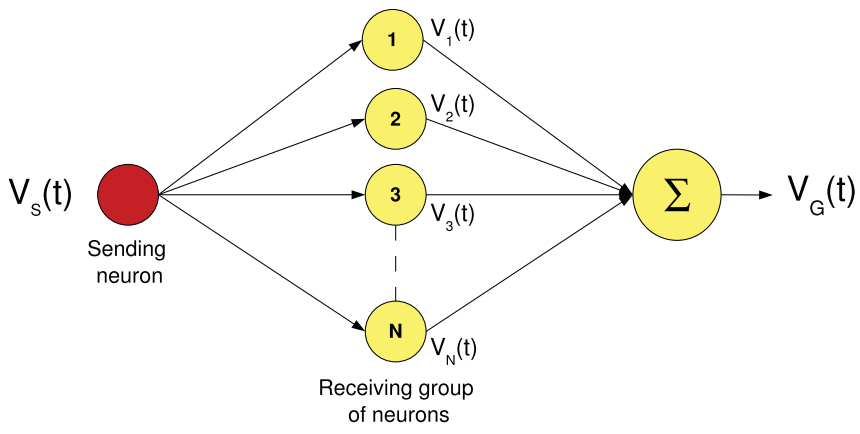

FIG. 7. (Color online) Group of $N$ parallel identical postsynaptic neurons coupled unidirectionally to the sending neuron. Entropy and mutual information are calculated for variables $V_{S}(t)$, train of spikes of the sending presynaptic neuron, and $V_{G}(t)$, summed over all individual $V_{i}(t)$ receiving postsynaptic trains of spikes.

of identical receiving neurons. The synaptic conductance $k$ is the same for all neurons in the group. The postsynaptic neurons are not mutually coupled, and the output $V_{G}(t)$ of the group is the sum over all individual postsynaptic spike trains $V_{i}(t), i=1, \ldots, N$. Entropy and mutual information are calculated for variables $V_{S}(t)$ and $V_{G}(t)$. The active sending presynaptic neuron is always activated with a random Gaussian current $I_{\text {stimulus }}$, supposedly induced by an external stimulus, what makes the neuron fire at a mean firing rate of $44 \mathrm{~Hz}$. Each of the postsynaptic neurons are exposed to a total noise current $I_{\text {noise }}$, modeled by a Gaussian noise of mean $0 \mu \mathrm{A} / \mathrm{cm}^{2}$ and variance $1 \mu \mathrm{A} / \mathrm{cm}^{2}$. These noise currents are independent for each neuron. In this section we present results relative to information transmission, energy consumption, and energy efficiency in the transmission for groups of $1,5,10$, and 20 neurons. The fact that the sending element can also be a group of neurons rather than a single neuron is irrelevant to our study.

\section{A. Information}

In this work we have performed a naive estimate [25] of the entropy of a train of spikes by generating successive windows of spikes of $75 \mathrm{~ms}$ length that are partitioned into five bins of $15 \mathrm{~ms}$ length each. The presence or absence of a spike inside one of these bins is codified as 1 or 0 , respectively, so that each window of spikes represents a symbol from a binary alphabet of 32 different symbols. If spikes from multiple neurons occur in the same time bin, this is treated as a single spike. To estimate entropies we have generated 5000 samples. Let $T_{1}^{i}$ and $T_{2}^{i}$ represent symbols of the grammar of the different possible symbols that can be coded with the spike train $V_{S}(t)$ of the presynaptic neuron and the output $V_{G}(t$ of the postsynaptic group of neurons, respectively. The mutual information, $I_{m}$, between the two variables can be defined as

$$
\begin{aligned}
I_{m}= & -\sum_{i} p\left(T_{2}^{i}\right) \log _{2} p\left(T_{2}^{i}\right) \\
& +\sum_{j} p\left(T_{1}^{j}\right) \sum_{i} p\left(T_{2}^{i} / T_{1}^{j}\right) \log _{2} p\left(T_{2}^{i} / T_{1}^{j}\right),
\end{aligned}
$$

that is, the entropy of the postsynaptic group minus the average of its conditioned entropies [26]. 


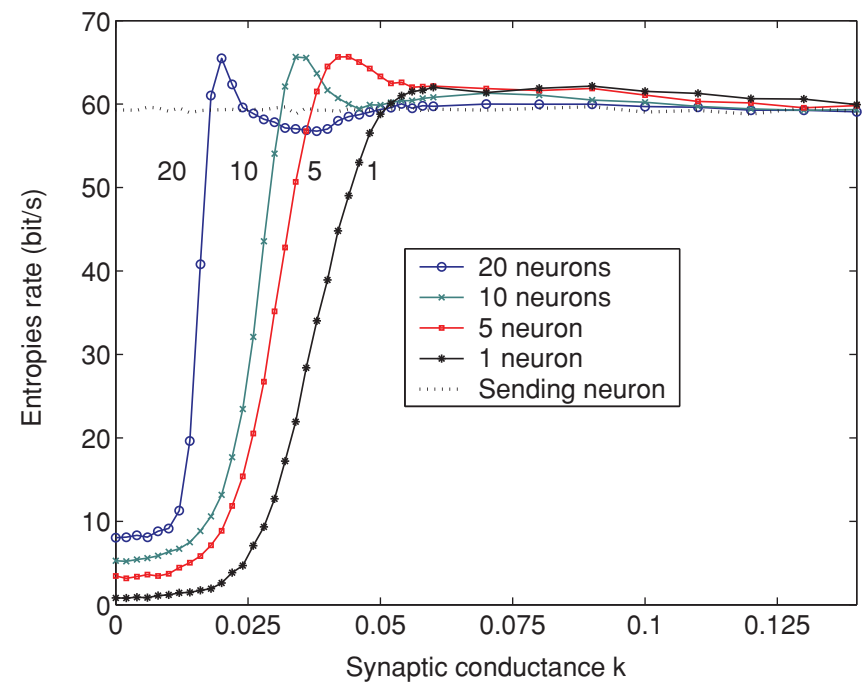

FIG. 8. (Color online) Entropies of the presynaptic sending neuron and the postsynaptic receiving groups of neurons at different values of the synaptic conductance $k$. Groups are of $1,5,10$, and 20 parallel identical postsynaptic neurons. The synaptic conductance $k$ is the same for all the neurons in the group. The stimulus current $I_{\text {stimulus }}(t)$ of the presynaptic neuron is a Gaussian noise of mean $0 \mu \mathrm{A} / \mathrm{cm}^{2}$ and variance $9 \mu \mathrm{A} / \mathrm{cm}^{2}$. Each of the postsynaptic neurons is exposed to a Gaussian noise current $I_{\text {noise }}$ of mean $0 \mu \mathrm{A} / \mathrm{cm}^{2}$ and variance $1 \mu \mathrm{A} / \mathrm{cm}^{2}$.

The calculated entropies of the sending and the receiving groups of neurons at different values of the synaptic conductance $k$ have been depicted in Fig. 8. As can be seen, the sending entropy is not affected by the strength of the unidirectional coupling and remains constant at rates of about 60 bits per second. For a single postsynaptic neuron its entropy steadily increases with the synaptic conductance $k$, reaches the entropy of the sending neuron at $k=0.05 \mathrm{mS} / \mathrm{cm}^{2}$, and after a very small overshooting stabilizes slowly at the same entropy of the presynaptic neuron. This is so because when uncoupled, at $k=0 \mathrm{mS} / \mathrm{cm}^{2}$, the postsynaptic neuron is near its quiescent state perturbed only by the occasional arrival of random excitation from some surrounding neurons. Under these conditions its entropy is near zero. As the coupling strength is increased, the firing rate of the postsynaptic neuron becomes progressively closer to the presynaptic firing rate and its entropy increases. For more than one postsynaptic neuron, activation of the postsynaptic entropy is more abrupt and reaches its maximum value at lower values of the synaptic conductance. For the groups of 5, 10, and 20 neurons the entropy of the sending neuron is reached at values of $k$ close to $k=0.035,0.03$, and $0.018 \mathrm{mS} / \mathrm{cm}^{2}$, respectively.

As a measure of the transmission of information Fig. 9 shows mutual information as function of the coupling strength $k$. For all the groups the mutual information increases slower than the entropy observed before. Thus, at values of $k=$ $0.05 \mathrm{mS} / \mathrm{cm}^{2}$, when a single postsynaptic neuron reaches its full entropy, the mutual information still has a long way to go. This means that at this coupling strength the postsynaptic neuron responds to the excitatory signals in its full variability but not in a sufficiently correlated manner. The conversion of the entropy observed in the receiving group of neurons into

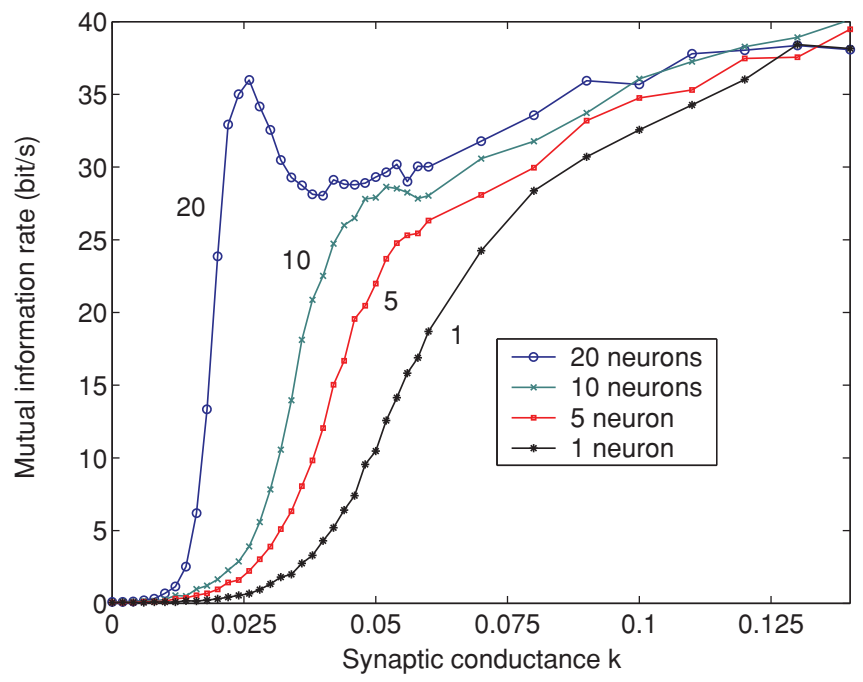

FIG. 9. (Color online) Mutual information between the sending and the groups of receiving neurons at different values of the coupling strength $k$. Groups are of 1, 5, 10, and 20 parallel identical postsynaptic neurons. Conditions are the same as those of Fig. 8.

mutual information depends on how much of the entropy of the group of neurons is dependent on the sending signal. If the channel is very noisy, in our experiment if neurons are poorly synchronized, the actual transmission of information will be relatively small compared with the observed entropy. In fact, complete synchronization between presynaptic and postsynaptic neurons is never reached, and the communication channel remains always noisy.

As Fig. 9 also shows, the groups of receiving neurons perform better than the single neuron. The improvement is very significant as the groups of neurons bring the ability to transmit information forward to lower values of the synaptic conductance $k$ and also reach higher relative maxima of mutual information. For the groups of 10 and 20 neurons their relative maxima of mutual information are clearly visible at values of the coupling near $k=0.05$ and $0.025 \mathrm{mS} / \mathrm{cm}^{2}$, respectively. As we will see, this fact has important implications for the energy efficiency of the transmission because the energy consumption of weak couplings is low.

\section{B. Energy}

In parallel with the information measures we have computed the average amount of metabolic energy consumed by the neurons at different values of the synaptic conductance $k$. The results are shown in Fig. 10. By metabolic energy consumption we mean, for a neuron, the sum over the three ion terms of Eq. (7) of the channels' consumption and for a group of neurons its accumulation to all the neurons in the group. This energy must be metabolically provided to the pumps, by hydrolysis of ATP molecules, to maintain the ion concentrations and thus the signaling activity of the neurons. For each of the different groups of neurons its pattern of consumption of energy is basically similar at a different scale. Ten neurons consume 10 times one neuron and half the consumption of 20. The constant metabolic energy consumption in the middle of the figure corresponds 


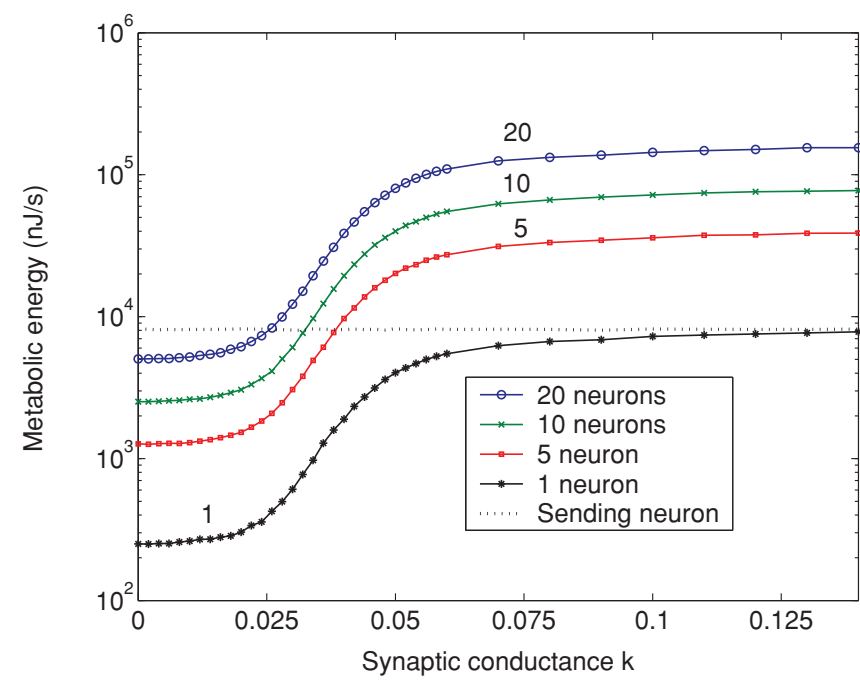

FIG. 10. (Color online) Average energy consumption of the ionic channels of the sending and the receiving groups of neurons at different values of the synaptic conductance $k$. Groups are of 1 , 5,10 , and 20 parallel identical postsynaptic neurons. The synaptic conductance $k$ is the same for all the neurons in the group. The stimulus current $I_{\text {stimulus }}(t)$ of the presynaptic neuron is a Gaussian noise of mean $0 \mu \mathrm{A} / \mathrm{cm}^{2}$ and variance $9 \mu \mathrm{A} / \mathrm{cm}^{2}$. Each of the postsynaptic neurons is exposed to a Gaussian noise current $I_{\text {noise }}$ of mean $0 \mu \mathrm{A} / \mathrm{cm}^{2}$ and variance $1 \mu \mathrm{A} / \mathrm{cm}^{2}$.

to the sending neuron. The signaling regime of this neuron is not affected by the synaptic conductance, and the stochastic variability of the random current $I_{\text {stimulus }}$ does not produce a detectable change in the average consumption of energy.

The average metabolic consumption of the sending neuron is about $9000 \mathrm{~nJ} / \mathrm{s}$, which according to our calculation of the efficiency per ATP in Sec. II of $0.39 \mathrm{eV}$ per ATP means that the metabolic consumption of energy of the sending neuron is $1.4 \times 10^{14}$ ATP per second and $\mathrm{cm}^{2}$ of membrane. Considering that the sending neuron fires at firing rates close to $44 \mathrm{~Hz}$ (see Fig. 5) it makes a metabolic consumption of about $31 \times 10^{11}$ ATP per $\mathrm{cm}^{2}$ of membrane to generate a single action potential. This result is 10 times greater than the $3.19 \times 10^{11}$ ATP per $\mathrm{cm}^{2}$ reported by Alle et al. [4] to generate an action potential in the rat mossy fiber at $37^{\circ} \mathrm{C}$ but considerably close to the $29 \times 10^{11}$ ATP per $\mathrm{cm}^{2}$ deduced from the original data reported by Hodgkin and Huxley [11] for the squid giant axon at $6.3^{\circ} \mathrm{C}$. The difference with the data by Alle et al. is attributable to the high extent of the overlap of the ion currents of the squid axon shown in Fig. 2.

As can also be seen in Fig. 10 the average consumption of energy per second of the postsynaptic groups is very dependent on the conductance $k$ of the synaptic coupling. At small values of the conductance $k$ the metabolic energy consumption remains relatively low. Between $k=0.02$ and $0.06 \mathrm{mS} / \mathrm{cm}^{2}$ the slope of the curves changes to a rapid increase of the metabolic energy consumption with the synaptic conductance. In this interval of $k$ values the metabolic consumption growths by a factor of 60 . The inflexion point corresponds to about $k=0.04 \mathrm{mS} / \mathrm{cm}^{2}$. As the metabolic energy consumption is extensive with the number of neurons, this pattern is similar for the different groups. We can also see in the plot that at low conductance values even the joint consumption of 20 postsynaptic neurons remains below the consumption of one single presynaptic signaling neuron.

On the other hand, at large values of the synaptic conductance the signaling regime of the group of 20 neurons requires about $1.2 \times 10^{5} \mathrm{~nJ}$ or $1.92 \times 10^{15} \mathrm{ATP}$ molecules per second and $\mathrm{cm}^{2}$ of membrane. This is a great consumption of metabolic energy. Nevertheless, as is shown in Fig. 9, a group of 20 postsynaptic neurons is able to retrieve information at low values of the synaptic conductance, which, as we will see in the next section, translates to a substantial optimization of the transmission energy efficiency.

\section{Information to energy consumption ratio: Energy efficiency}

This section studies the energy efficiency of the transmission of information at different values of the conductance $k$. The results for the different groups of neurons are presented in Fig. 11 as the ratio of mutual information rate to metabolic energy consumption. In all cases energy consumption refers to the total energy involved in the process, that is, the sum of the metabolic energy consumed by the presynaptic and all postsynaptic neurons.

One single neuron exhibits a monotonic sigmoid growth of its information to energy ratio as the synaptic conductance $k$ increases. Better efficiency in bits per unit of energy requires larger values of the synaptic conductance $k$ and so greater values of metabolic energy consumed per second, as shown in Fig. 10. There is no compromise; any attempt to transmit information at lower values of $k$, will eventually lead to a greater consumption of energy for the transmission of that information. As can be seen in Fig. 11 the single neuron reaches is best performance at values of the synaptic conductance at about $k=0.14 \mathrm{mS} / \mathrm{cm}^{2}$, which is the largest shown. At this coupling conductance the cost of the information is 0.175 bits $/ \mathrm{nJ}$, which means $9 \times 10^{6}$ ATP molecules to transmit one bit of information. As said, this cost also includes the presynaptic neuron; that is, half of it, about $4.5 \times 10^{6}$ ATP

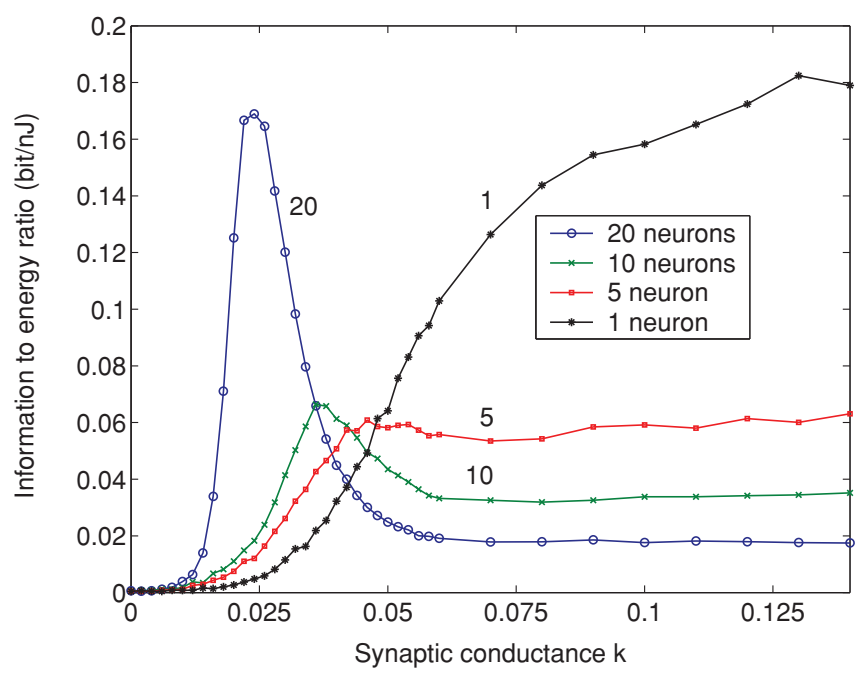

FIG. 11. (Color online) Mutual information to energy consumption ratio for the groups of neurons at different values of the coupling strength $k$. The energy consumption corresponds to the total energy consumed by the sending neuron and the group of receiving neurons. 
molecules per bit would be the cost of the postsynaptic neuron. This result is in good agreement with reported values between $9 \times 10^{5}$ and $3.0 \times 10^{6}$ ATP molecules per bit for the cost of information transmitted by spikes of cells in the blowfly retina [27]. The discrepancy is consistent with the large overlap of the ion currents of the Hodgkin-Huxley model at $6.3{ }^{\circ} \mathrm{C}$.

The pattern described for a single neuron changes for a group of postsynaptic neurons. The monotonic sigmoid growth of the information to energy ratio no longer applies, and, for each studied group, a relative maximum of this ratio appears. As can be appreciated, as the size of the group increases, the maxima take place at progressively weaker couplings and also become sharper and higher. The group of 20 neurons display a sharp maximum at $k=0.022 \mathrm{mS} / \mathrm{cm}^{2}$, reaching nearly the best performance of the single neuron. This sharp maximum supposes the best compromise between information and energy consumption for the group of 20 neurons. At this value of the synaptic conductance the metabolic energy consumption of the group is $9000 \mathrm{~nJ} / \mathrm{s}$, about 12 times lower than its maximum consumption at $k=0.14 \mathrm{mS} / \mathrm{cm}^{2}$.

The fact that for groups of postsynaptic neurons there are values of the synaptic conductance that optimize the energy efficiency of the transmission of information is one of the main findings of this paper. For a group of neurons information grows with $k$ much faster than energy consumption at low values of the conductance and much slower at large values, which produces relative maxima in the ratio. One key aspect for this is that the group simply adds all the induced responses of each individual neuron, producing a joint received signal that retains the valuable information contained in each of the individual signals. Noise also helps to improve the cooperative behavior of the group [28,29]. Thereby, the group retrieves from the presynaptic train of spikes the same levels of information a single neuron would retrieve but is able to do it at lower values of synchrony than a single neuron would do. As a consequence information is obtained at relatively low levels of metabolic energy consumption.

\section{DISCUSSION AND CONCLUSIONS}

\section{A. The energy approach}

This paper discusses a different approach to evaluating the metabolic energy consumption of a neuron to maintain its signaling activity and uses it to estimate the metabolic cost of transmitting information between neurons. Biophysical considerations about the nature of the Hodgkin-Huxley model have guided us to find an analytical expression of the electrochemical energy involved in the dynamics of the model. The time derivative of this energy gives the balance of energy consumption and provision during its signaling behavior. To our knowledge, apart from some previous attempts to find energy functions for neuron models with chaotic dynamics [30-32], this approach has not been used in the literature. The Hodgkin-Huxley model is both of wide interest and very appropriate for investigating energy aspects since it is based on sound biophysical principles.

In the Hodgkin-Huxley model there is no explicit representation of the ionic pumps, which are essential in the evaluation of the metabolic energy consumption of the neuron. The circuit describes a quasi-steady state immediately after a hypothetical shutdown of the ion pumps [20]. In the model, the batteries representing the Nernst potentials provide the energy to keep them constant, while in the real neuron this energy is provided by the ion pumps. If the batteries do not go flat in the model, it means that the ion pumps are working in the real neuron. Thus, the dissipation of energy in the variable resistors of the circuit represents the loss of electrochemical energy of the ions, which must be continuously replenished by the batteries, that is, by the ion pumps.

This approach has two clear advantages. First, the model describes the actual dynamics of the squid giant axon and the energy involved without requiring any priory hypothesis about the stoichiometry of the ions or the extent of the overlapping. Estimating energetic costs of other neuron types requires developing specific models. A second advantage of the approach is that it works in complex networks of neurons taking into account all the interactions. As commented in Ref. [33] it is not possible to estimate energetic costs in isolation. As far as possible the complexity of the interactions should be taken into account.

\section{Experimental support}

The values of energy consumption, energy delivered per ATP molecule, and number of ATP molecules required to transmit a bit of information that our model has produced are consistent with relevant experimental data in the literature. To pump one sodium out of the cell costs both electrostatic potential energy and the free energy of increasing the difference in sodium concentrations across the membrane. The total cost of one pump cycle, which pumps three sodium ions out of the cell and two potassium ions in, can be estimated to be $0.37 \mathrm{eV}$ [20]. On the other hand, one ATP molecule hydrolyzed under normal physiological conditions liberates about $0.47 \mathrm{eV}$ [20] or $0.43 \mathrm{eV}$ [21]. Our results with the Hodking-Huxley model [see Fig. 3(d)], are in remarkable agreement with these estimates. In the firing regime, our computation demands to each ATP molecule $0.39 \mathrm{eV}$ of liberated free energy. This agreement implies that our method of calculation of the actual energy consumption by the pump and the number of ATP molecules involved are consistent with other data in the literature and also that the Hodgkin-Huxley model produces very accurate estimates of energy consumption.

We have also found that in the model the metabolic cost per bit retrieved by a single neuron decreases with the synaptic conductance until a saturation value of $4.5 \times 10^{6}$ ATP molecules per bit. This result, considering the overlap in the ion currents of the Hodgkin-Huxley neuron, is in reasonably good agreement with reported data of costs between $9 \times 10^{5}$ and $3 \times 10^{6}$ ATP molecules per bit for information transmitted by spikes of cells in the blowfly retina [2].

According to our work the fraction of sodium ions that has to be considered to estimate the number of ATP molecules to generate an action potential is one third [Fig. 3(d)]. This result agrees with the majority opinion in the literature and supports the possibility that sodium ions remain linked to the dynamics of the enzyme ATPase while potassium ions are free to participate in processes different from the generation of action potentials. These processes would compensate the 
unbalance left by the pumps in the moving back of ions to restore the ions' concentrations after action potentials.

\section{B. Information and synchronization}

Transmitted information between neurons has been measured by mutual information between trains of spikes from presynaptic and postsynaptic neurons. The source of information entropy is a random stimulus current that the sending neuron perceives and that it translates into spike trains. Estimates of mutual information have been made based on its primary definition without a particular hypothesis about the structure of the signals. Trains of action potentials have been converted into temporal sequences of binary code, and the required absolute and conditioned probabilities expressed in Eq. (9) have been computationally estimated. This naive procedure is computationally heavy and imposes limitations on the resolution of the temporal localization of the spikes and to the maximum size of the binary words to use. We have analyzed the output of the postsynaptic group without any kind of preprocessing limiting the experiment to group sizes computationally tractable by our algorithm. Large numbers of neurons, for example, several hundred, produce practically graded informational output, and a different style of algorithm to estimate mutual information is required [34].

In the transmission of information synchronization seems to play a key role. Veruky and Hartveit [35] show that spike generation between pairs of AII amacrine cells can be synchronized precisely and that there is evidence that spikes can be transmitted through electrical synapses between these cells. Also, in Ref. [18] they demonstrate temporally precise synchronization of subthreshold membrane potential fluctuations between amacrine cells and $\mathrm{ON}$-cone bipolar cells. The degree of synchrony reached by unidirectionally coupled neurons typically increases with the coupling strength, that is, with the conductance $k$ of the electrical gap junction. Thereby, we have studied both energy and information between coupled neurons at different values of the coupling conductance $k$.

\section{Information and energy: Energy efficiency}

As groups of neurons acting cooperatively are efficient to retrieve information and frequent in many regions of the brain $[23,24,28,29]$, we have studied the transmission of information between one single sending neuron and groups of receiving neurons, which produce as output the sum over all the individually generated trains of actions potentials. We have found that when the receptor is a single neuron the mutual information increases monotonically with $k$ faster than the metabolic energy consumption rate, which shows that the most energy-efficient condition occurs at the saturation level. For a single receiving neuron there is no optimal compromise. On the contrary, for groups of receiving neurons, there are relatively small values of the synaptic conductance $k$ that optimize the ratio of mutual information to metabolic energy consumption. Working at these coupling conditions is for the group of neurons the most energy-efficient option. Note that at these low values of the synaptic conductance each node in the network carries out lossy compression on its input and the neural network models an stochastic pooling system in the sense defined by McDonnell et al. [36]. Each neuron in the group communicates compressed measurements at very low energy cost. The outputs of each neuron are combined to form an overall network output that causes no further loss of information about the presynaptic signal, when compared with the best that could be obtained if all postsynaptic individual signals were available. Thus, 20 postsynaptic neurons make a more robust and reliable channel than a single neuron, and they work jointly at the same metabolic cost per unit time a single neuron does. It seems likely that real neurons use robust efficient circuits of this type to generate and transmit information.

It has been reported [37] that the neural organization observed in the early visual system is compatible with an efficient use of metabolic energy. The central surrounding organization of retinal ganglion cells optimizes the use of energy when encoding natural images. Other aspects of the organization such as the higher densities of receptive fields in the fovea that decrease in the periphery could also be an attempt to optimize the use of metabolic energy [37]. In order to test their energy-efficient coding hypothesis Vincent et al. [37] use a model where the metabolic cost of the synaptic activity and firing rates can be fully manipulated. In the retinal stage, a cost that increases in proportion to synaptic activity is imposed, while in the cortical stage they suppose a cost proportional to the firing rate of neurons. Although this is certainly a plausible hypothesis, it is not based on any comprehensive model of energy linked to the true dynamics of the firing regime of the neurons.

Also, energy-efficient information transmission from the point of view that inputs are optimally encoded into Boltzmann distributed output signals has been analyzed in Ref. [38]. An open question is the determination of the energy cost of generating the spike trains that codify each of the different output symbols. Our approach could provide a way to determine the energy cost of the generation of these spike trains, and, in general, we think that models of energy such as the one described in this paper could provide support to empirical studies to ascertain if neurons really are taking advantage of efficiency savings.
[1] D. Attwell and S. B. Laughlin, J. Cerebr. Blood Flow Metab. 21, 1133 (2001).

[2] S. B. Laughlin, Curr. Opin. Neurobiol. 11, 475 (2001).

[3] P. Siekevitz, Science 306, 410 (2004).

[4] H. Alle, A. Roth, and J. R. P. Geiger, Science 325, 1405 (2009).
[5] A. L. Hodgkin, Phil. Trans. R. Soc. B 270, 297 (1975).

[6] D. Attwell and C. Iadecola, Trends Neurosci. 25, 621 (2002).

[7] P. Lennie, Curr. Biol. 13, 493 (2003).

[8] B. Sengupta, M. Stemmler, S. B. Laughlin, and J. E. Niven, PLoS Comput. Biol. 6, e1000840 (2010). 
[9] P. Crotty, T. Sangrey, and W. B. Levy, J. Neurophysiol. 96, 1237 (2006).

[10] W. D. Stein, Int. Rev. Cytol. 215, 231 (2002).

[11] A. L. Hodgkin and A. F. Huxley, J. Physiol. 117, 500 (1952).

[12] R. H. Adrian, Proc. R. Soc. B 189, 81 (1975).

[13] A. Destexhe, M. Rudolph, J. Fellous, and T. Sejnowski, Neuroscience 107, 13 (2001).

[14] F. Bezanilla, R. E. Taylor, and J. M. Fernandez, J. Gen. Physiol. 79, 21 (1982).

[15] T. D. Sangrey, W. O. Friesen, and W. B. Levy, J. Neurophysiol. 91, 2541 (2004).

[16] C. Shannon and W. Weaver, The Mathematical Theory of Communication (University of Illinois Press, Urbana, 1949).

[17] H. Kolb and E. Flamiglietti, Science 186, 47 (1974).

[18] M. L. Veruki and E. Hartveit, J. Neurosci. 22(24), 10558 (2002).

[19] W. Gerstner and W. Kistler, Spiking Neuron Models: Single Neurons, Populations, Plasticity (Cambridge University Press, Cambridge, UK, 2002).

[20] P. Nelson, Biological Physics (W. H. Freeman and Company, New York, 2004).

[21] Z. Sinkala, J. Theor. Biol. 241, 919 (2006).

[22] E. Garcia-Perez, M. Vargas-Caballero, N. Velazquez-Ulloa, A. Minzoni, and F. F. De Miguel, Biophys. J. 86, 646 (2004).

[23] M. D. McDonnell and N. Stocks, Scholarpedia 4, 6508 (2009).

[24] N. G. Stocks, Phys. Rev. Lett. 84, 2310 (2000).
[25] S. P. Strong, R. Koberle, R. R. de Ruyter van Steveninck, and W. Bialek, Phys. Rev. Lett. 80, 197 (1998).

[26] F. Rieke, D. Warland, R. de Ruyter van Steveninck, and W. Bialek, Spikes: Exploring the Neural Code (MIT Press, Cambridge, MA, 1999).

[27] S. B. Laughlin, R. R. de Ruyter van Steveninck, and J. C. Anderson, Nat. Neurosci. 1, 36 (1998).

[28] T. Hoch, G. Wenning, and K. Obermayer, Phys. Rev. E 68, 011911 (2003).

[29] S. Wang, F. Liu, W. Wang, and Y. Yu, Phys. Rev. E 69, 011909 (2004).

[30] F. J. Torrealdea, A. d'Anjou, M. Graña, and C. Sarasola, Phys. Rev. E 74, 011905 (2006).

[31] F. J. Torrealdea, C. Sarasola, and A. d'Anjou, Chaos Soliton Fract. 40, 60 (2009).

[32] F. J. Torrealdea, C. Sarasola, A. d'Anjou, A. Moujahid, and N. V. de Mendizabal, Biosystems 97, 60 (2009).

[33] G. Buzsaki, K. Kaila, and M. Raichle, Neuron 56, 771 (2007).

[34] J. E. Niven, J. C. Anderson, and S. B. Laughlin, PLoS Biol. 5, 0828 (2007).

[35] M. Veruki and E. Hartveit, Neuron 33, 935 (2002).

[36] M. D. McDonnell, P.-O. Amblard, and N. G. Stocks, J. Stat. Mech. (2009) P01012.

[37] B. Vincent, R. Baddeley, T. Troscianko, and I. Gilchrist, Network: Comput. Neural Sys. 16, 175 (2005).

[38] V. Balasubramanian, D. Kimber, and M. J. Berry II, Neural Comput. 3, 799 (2001). 